\title{
The Calibrated Automated Thrombogram (CAT): a universal routine test for hyper- and hypocoagulability
}

\author{
H.C. Hemker'; P.Giesen', R. AIDieri', V. Regnault', E. de Smed', R. Wagenvoord', \\ T. Lecompte ${ }^{2}$ and S. Béguin ${ }^{1,2}$ \\ 'Synapse BV, Cardiovascular Research Institute Maastricht; ${ }^{2} \mathrm{CHU}$ de Nancy, Vandoeuvre France
}

\section{Key Words}

Thrombin; Thrombogram; hypercoagulability; hypocoagulability; thrombin generation test.

\section{Summary \\ By using a "slow" fluorogenic thrombin substrate and continuous comparison to a simultaneously run calibra- tor, thrombin generation can be monitored automatical- ly, on line, in clotting PPP or PRP at a throughput of up to 100 samples per hour. The resulting "Thrombogram" in PPP measures hypocoagulability (haemophilias, oral anticoagulants, heparins (-likes), direct inhibitors) and hypercoagulabilities (AT deficiency, prothrombin hyper- expression, prot.C and S deficiency, factor V Leiden, oral contraceptives). \\ In PRP it is diminished in thrombopathies, in von Willebrand disease, by antibodies blocking GPIIb-IIla or GPIb, or by antiplatelet drugs like aspirin and clopido- grel. Lupus anticoagulant both retards and increases thrombin generation. The thrombogram thus appears to be a broad function test of the haemostatic- thrombotic mechanism of the blood.}

Copyright @ 2002 S. Karger AG. Basel

\section{Introduction}

Disorders of the haemostatic thrombotic system (HTS) are pivotal in over half of all invalidating and lethal disease. Quantitatively the less important: haemophilia and lung embolism, come readily to mind. That arterial thrombosis causes coronary infarction and stroke are essentially thrombotic diseases is less often acknowledged. That one out of ten of the elderly risk loss of brain function through embolisation on basis of atrial fibrillation and carotid emboli, or that victims of serious accidents and patients suffering from sepsis risk fatal intravascular coagulation is not so widely acknowledged. More people die from arterial thrombosis than from malignancies and more from venous thrombosis than from accidents. In view of such medical importance it is strange to recognize that there is no valid function test of the HTS available to the clinician [1].

Clotting times are the best we have and they are insensitive to mild haemostatic disorders (e.g. carriers of haemophilia, mild liver disease) or to increased coagulability that leads to increased thrombosis risk. Clotting tests often need to be adapted to a specific use. To give one example, the thromboplastin time (=Prothrombin time $=$ Quick time) can be used for diagnosis of serious liver disease or treatment with anticoagulants but is not prolonged by haemophilia or heparin treatment. Much of the art and science of the clinical coagulation lab resides in knowing how to interpret the scattered information that can be get out of clotting times of different types, platelet aggregation, bleeding time etc.

\section{KARGER \\ Fax +4161306 1234 \\ E-Mailkarger@karger.ch}

www.karger.com (c) 2002 S. Karger AG. Basel

$1424-8832 / 02 / 0326-0249 \$ 18.50 / 0$

Accessibile online at: www.karger.com/journals/pht
Prof. Dr. .H.C.Hemker

Synapse b.v. CARIM,

P.O.Box 616 6200MD Maastricht, The Netherlands.

Tel: 0031-43-3881675; Fax: 0031-43-3884578.

E-Mail: HC.Hemker@thrombin.com. 
The insufficiency of the over-all tests is partly compensated for by a great variety of sophisticated tests of single components of the clotting system, so many indeed that a judicious selection should be made in every special case, which is the other half of the specific knowledge of the clinical haemostasis lab.

The combination of unsatisfying general function tests and a broad spectrum of specific test evidently leaves room for a general function test of the HTS. The thrombin generation test has been known for a long time to be a valid physiological function test of the clotting system [2,3]. That it never has been used on any significant scale to probe haemostatic function in the clinics, in epidemiology and in the search for new antithrombotic drugs is due to the fact that with the conventional (subsampling) techniques, one curve requires about one man-hour of skilled laboratory work. In this article we present a technique, the calibrated automated thrombogram (CAT), that allows to obtain $\sim 100$ thrombin generation curves per man-hour, without requiring ultra-sophisticated equipment.

\section{Thrombin}

Thrombin takes a focal place in the mechanism of haemostasis and thrombosis [4], in that, on the one side, scores of factors influence its formation and on the other side it has numerous actions on blood and vessel components. In the haemostatic mechanism there are no pathways that bypass thrombin. Thrombin generation therefore reflects much - if not all - of the thrombotic-haemostatic function of the blood. This has been recognised all through the history of haemostasis research. Thrombin generation is far from being a novel technique. In the older literature extensive validation of this test can be found both as diagnostic aid and as a means for (patho-)physiological research. More recently, using subsampling methods, it has been shown that thrombin generation in platelet poor plasma (PPP) is diminished in all types of coagulation factor deficiency and anticoagulant treatment (oral, heparin (-like), direct thrombin inhibitors) In platelet rich plasma (PRP) it is diminished in congenital and acquired platelet related disorders (e.g. Glanzmann's thrombasthenia, von Willebrand disease) and by antiplatelet drugs (Aspirin, blockers of GPIIb-IIIa, clopidogrel). On the other hand thrombin generation in PPP is increased in congenital and acquired thrombophilias (AT deficiency, hyperprothrombinaemia, deficiencies of protein $\mathrm{S}$ or $\mathrm{C}$, oral contraceptives). For references see ref 1. Thrombin generation in PRP also allows to observe hitherto often overlooked interactions between platelets and the clotting system. It has been shown e.g. that vWF and fibrin play a crucial role in normal thrombin generation in PRP [5]. A typical course of thrombin formation in clotting blood or plasma (the Thrombogram ${ }^{\circledR}$ ) is shown in figure 1. After a period in which no observable thrombin is formed, the con- centration steeply goes up, rises to a peak and then goes down again. The parameters are the lag time, the area under the curve (AUC=ETP, see below), the peak height, and the time it takes to reach the peak.

\section{Methods to determine the thrombogram or one of its parameters}

In a way one can say that measuring a clotting time is a way to obtain one parameter of the thrombogram, i.e. the lag time of thrombin formation. Fibrin appears as soon as the first traces of thrombin form and for all practical purposes the end of the lag time is the moment at which clotting occurs. It is also the most whimsical of the thrombogram parameters, which explains limited use of clotting times as a function test.

The endogenous thrombin potential (ETP) can be obtained by adding a thrombin substrate in excess, so that the total amount of substrate converted is proportional to the area under the thrombogram. Two such methods have been published, one using artificial, slow thrombin substrates and measuring the end level of split product [6, 7], the other measuring the amount of $\alpha 2$ macroglobulin bound thrombin $(\alpha 2 \mathrm{M}-\mathrm{T})$ that is found after the thrombin generation process has taken place in defibrinated plasma [8].The use of slow artificial substrates is limited by the requirement that, in order for the amount of product to reflect truthfully the ETP, unrealistically high concentrations of chromogenic substrate must be used, unless additional antithrombin is also added (see also below) [6]. In the $\alpha 2 \mathrm{M}-\mathrm{T}$ method, defibrination is required because in the presence of fibrin the amount of thrombin that reacts with $\alpha 2$ macroglobulin is minimal. The outcome remains dependant upon the $\alpha 2-\mathrm{M}$ level of the plasma and the defibrination procedure. The method has been used with advantage in research on the hypercoagulability induced by oral contraceptives [9].

Thrombogram like curves can also be obtained by monitoring the capacity of a clot to mechanically connect two surfaces, as in thromboelastography [10] or by measuring the development of turbidity due to clot formation [11]. Both methods are perfect for measuring the clotting time. Thromboelastography has the additional advantage of using whole blood. The disadvantage of such methods is that the dependant variable (mechanical displacement, turbidity) is not a conceptual entity that is directly related to haemostasis and thrombosis, like thrombin concentrations are, but a covariable of one of the effects of thrombin formation, i.e. fibrin (-monomer) polymerisation; while this polymerisation itself is related to thrombin formation, or even to the formation of fibrin monomers, in a complicated and as yet incompletely understood manner.

Such methods are dependant both on thrombin formation and on the quantity and properties of fibrinogen. In cases where fibrinogen increases but thrombin generation decreases 
(e.g. a patient with fever under anticoagulant treatment) this will cause ambiguous results. The fundamental difference between such methods and the Thrombogram is that an effect is studied that is dependant in an unknown way on known and unknown variables that may or may not be relevant to the state of the patient. The complete Thrombogram is traditionally obtained via subsampling from clotting blood or plasma at timed intervals and measuring the concentration of thrombin in each sample $[3,5,12]$.

Continuous monitoring of thrombin concentration has been attempted through adding a suitable thrombin substrate to the clotting sample and monitoring the time course of appearance of the amidolytic split product. E.g. a chromogenic substrate is used and the optical density is measured so as to monitor the development of p-nitro-aniline [7].

For a number of reasons in such methods the velocity with which the output signal (OD, fluorescence) appears is not dependant upon thrombin concentration only, so that the slope of the product curve does not directly represent the amount of thrombin present. The causes are:

A: Substrate depletion: The signal is dependent upon the concentration of substrate which, through the very enzyme activity itself, decreases in time. The effect can be attenuated by adding an excess of substrate but to a certain limit only. The substrate binds, reversibly, to the active centre of thrombin and thereby protects thrombin from inactivation by natural antithrombins. Abolishing the effect of substrate consumption to an acceptable degree is paid for by prolonging the experiment to last for about two hours. Also, at such concentrations of substrate, thrombin inhibition interferes with feedback reactions and it is no longer guaranteed that the natural process is measured. This is also the reason that, in a method meant to assess the area under the curve from the total amount of substrate converted, extra antithrombin needs to be added in order to make the experiment practically possible [6].

B: Changes in optical density occur through clotting of the plasma sample [11]. The use of chromogenic substrates implies the removal of fibrinogen, and consequently of blood platelets. Fibrinogen and platelets, however, are essential components of the clotting system that influence the course of thrombin formation (see below). This puts a serious limit on the applicability of optical density as a detection method. This problem can be circumvented by using a substrate that yields a fluorescent product [13]. This, however, introduces the next problem:

$\mathrm{C}$ : In fluorescence measurements the signal is not linearly dependent upon the concentration of fluorescent product, because fluorescent molecules absorb the light from other product molecules, the so called "inner filter effect". Increasing substrate concentrations, as required for limiting the effect of substrate consumption automatically increases the inner filter effect. Problem $\mathrm{A}$ is common to all continuous methods. Problem B can be circumvented by using a fluorogenic substrate but this introduces problem $\mathrm{C}$.
Because of $\mathrm{A}$ and $\mathrm{C}$ there is no constant factor by which the velocity of signal production must be multiplied in order to obtain the thrombin concentration. The calibration factor furthermore varies from experimental setup to experimental setup (e.g. is different in different fluorometers) and from sample to sample (e.g. due to color variations of the plasma). Addition of a known standard amount of thrombin to the sample is impossible because the enzyme added will disturb the physiological reactions and will be rapidly inactivated by antithrombins. We solved these problems through measuring the activity of a stable calibrator in a parallel non-clotting sample, as described in the next section.

\section{The Calibrated Thrombogram, differences with previous methods}

We realised that the effect of substrate consumption and the inner filter effect are both functions of the amount of product present and hence of the level of fluorescence. That means that the calibration "constant" is not in reality a constant but varies with the magnitude of the fluorescent signal (be it not linearly). Consequently at every level of fluorescence there is a unique calibration constant that gives the relation between the velocity of signal increase and thrombin concentration.

We determine this varying calibration constant in a parallel experiment in which a calibrator, i.e. a constant, known, thrombin like activity is added to a sample of the same plasma in which no thrombin generation is triggered. In this way variations due to the instrument and to the colour of the plasma are equalled out. As the calibrator consumes the substrate, it increases the fluorescence level and thus Kc can be measured over a range of fluorescence levels. As a calibrator we use the $\alpha 2$ macroglobulin-thrombin complex. Binding of thrombin to $\alpha 2$ macroglobulin renders thrombin immune to the natural inactivators present in plasma but leaves intact its capacity to split small thrombin substrates.

In practice we split a sample in two parts $(80 \mu \mathrm{L})$ and add substrate to both. In one sample coagulation is triggered by recalcification and a low concentration of tissue factor. To the other sample the calibrator is added. The signal from the two wells is simultaneously measured. From the calibrator well, the calibration constant is obtained at every level of fluorescence. With these values, the molar amount of thrombin present at any moment in the coagulating sample is calculated and rendered on the screen during the experiment by a computer program developed in our department (fig.1).

\section{Method}

We only give the outlines of the method, an article with detailed information is in preparation [14]. The experimental 
conditions are essentially those used previously with the subsampling method [13]. Human platelet rich- and platelet poor plasma (PPP/PRP) are prepared and platelets are counted on a Coulter counter. The thrombogram is relatively insensitive to the platelet count at values of $200.000 / \mu \mathrm{L}$ and above. If in vitro inhibitions are to be investigated, adjusting with autologous PPP to a low normal value $(150.000 / \mu \mathrm{L}$.) renders the experiment more sensitive to slight inhibitions. Buffer: $20 \mathrm{mM}$ Hepes, $140 \mathrm{mM} \mathrm{NaCl}, 5 \mathrm{~g} / \mathrm{l}$ bovine serum albumin (BSA), $\mathrm{pH}$ 7.35; with $0.02 \%$ sodium azide as a preservative. FluCa solution: Buffer with $60 \mathrm{mg} / \mathrm{mL}$ of BSA, $0.1 \mathrm{M} \mathrm{CaCl} 2$ and $2.5 \mathrm{mM}$ of Z-Gly-Gly-Arg-AMC (Bachem, Switzerland). Trigger solution for PRP: 3 pM recombinant tissue factor (rTF) in buffer, for PPP $30 \mathrm{pM}$ rTF and $24 \mu \mathrm{M}$ procoagulant phospholipids (PS,PC,PE) in buffer.

In a 96-well round bottom plate, $20 \mu \mathrm{L}$ of prewarmed trigger solution is added to one well and $20 \mu \mathrm{L}$ of prewarmed calibrator, i.e. $600 \mathrm{nM}$ alpha2M-thrombin complex to another; 80 $\mu \mathrm{L}$ of plasma is added to both wells. $\mathrm{Ca}^{++}$is added together with the substrate at zero time (Fluka Solution). Readings are done in a microtiter plate fluorometer (Fluoroscan Ascent, Thermolabsystems, Helsinki, Finland), at $37^{\circ} \mathrm{C}$, provided with a dispenser that is filled with FluCa solution. When the experiment is started, the dispenser squirts $20 \mu \mathrm{L}$ of FluCa solution in each well at zero time. On the screen of the computer appears a picture as in fig.1, i.e. the signal from the calibrator and the calculated thrombin concentration that is calculated from the signal of the well in which thrombin is generated and the calibration signal.

The displayed thrombin concentration is a preliminary value because $\alpha 2$-macroglobulin-thrombin builds up from the thrombin generating in the experiment and the $\alpha 2$-macroglobulin normally present in any plasma. This is a common feature of all thrombin generation measurements in which thrombin is

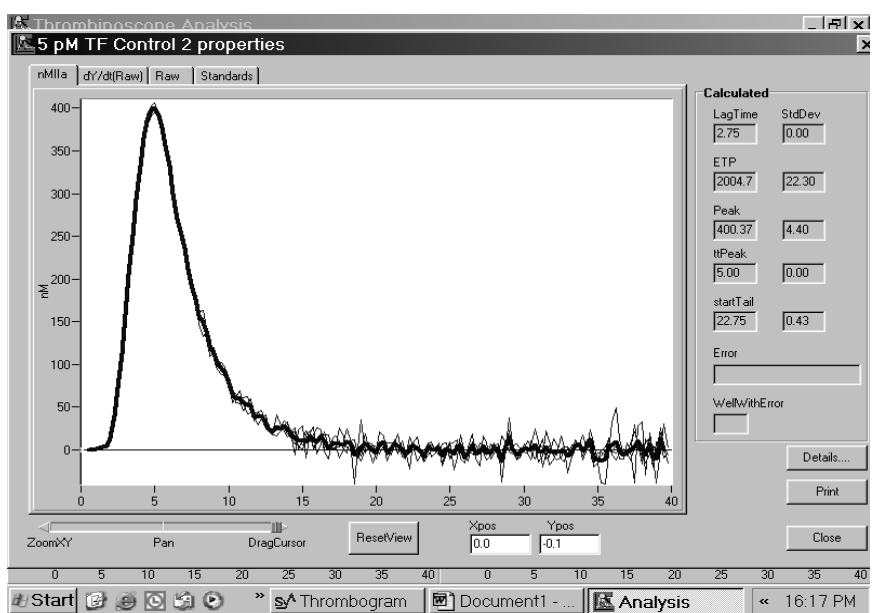

Fig. 1. Screen picture of an automated Thrombogram The thin lines represent the signal from four identical experiments. The bold line is the median of the four. estimated by its amidolytic activity on a small signal-substrate, subsampling and continuous methods alike. The way to correct for this effect has been published [15]. This correction is carried out by the software after the experiment. It should be mentioned that serious errors can be made if the effects of substrate consumption and inner filtering are compensated via the algorithm designed for $\alpha 2 \mathrm{M}-\mathrm{T}$ correction, even though the resulting curves resemble thrombin generation curves. Such curves can be easily obtained with any fluorometer but, lacking calibration, they represent semi-quantitative approaches to the Thrombogram.

The experiment shown in fig. 1 shows the superposed curves from 4 identical thrombin generation experiments together with 4 calibration experiments. The software allows to display any number of identical experiments in one frame. We advise to run each experiment (clotting plasma and calibrator) at least in duplo. Once in every 3 or 4 full plates a well with a bubble presents, resulting in an erratic curve. Such curves are easily recognised and can be removed via the software.

As each plasma minimally requires two clotting- and two calibration wells, up to 24 different plasmas can be measured in parallel. If a concentration series of a drug is tested in vitro on the same plasma, wells with different concentrations of inhibitor can share their calibrators. The only requirement being that always an experiment in a given plasma is accompanied by the registration of the signal from the standard in another sample of that same plasma. Experimental error $(\mathrm{CV}<$ $5 \%$ ), inter and intra-individual coefficients of variation and normal values will be discussed in a separate article [14]. It will be shown that experimental error and day to day variation in the same person are low $(\sim 5 \%)$ whereas interindividual variation is high.

\section{Results}

Several articles are in preparation to illustrate the use of the thrombogram in different conditions. Here we can only give a summary and some preliminary results.

Congenital clotting factor deficiencies.

Thrombograms confirm the results found previously with the subsampling method: In haemophiliac plasma (both haemophilia A and B) the thrombogram is low and can be normalised by infusion of the deficient factor but also, in the case of haemophilia A by DDAVP-infusion. Interestingly, infusion of factor VIIa also increases thrombin generation in haemophiliacs. From patients with rare clotting disorders (factors II, V, VII, X, XI), the same plasmas that were studied by subsampling by Aldieri et al. [16] were, for so far as available, tested with the automated technique and essentially identical results were found. In von Willebrand disease, the factor VIII deficiency is often high enough to ensure normal thrombin 
generation, so that a normal thrombogram is found in PPP but the defect in platelet activation becomes obvious when thrombin generation is tested in PRP.

\section{Platelet disorders and platelet drugs}

The defect in Glanzmann's thrombasthenia readily shows up, as does the blocking of GPIIb-IIIa by antibodies (Reopro) and other antagonists. Bernard-Soulier's disease is a special case that will be the subject of a forthcoming publication. The effects of aspirin and Clopidogrel are readily seen. The results obtained with a number of platelet agonists and antagonist show that the role of the platelet in blood coagulation is a sensitive probe for platelet function. Notably the lack of von Willebrand factor causes diminished generation of thrombin in PRP whereas an excess of this factor increases thrombin generation.

\section{Hypercoagulability}

Thrombin generation is the only test that is sensitive to hypercoaguable changes in the plasma [17] Increase of thrombin generation is observed in deficiency of antithrombin as well as in congenital hyperprothrombinaemia. When thrombomodulin is added, thrombin generation becomes sensitive to all congenital and acquired (oral contraceptives!) disorders of the protein $\mathrm{C}$ system. When thrombin generation plus and minus thrombomodulin are measured, an exact estimate of the function of the protein $\mathrm{C}$ system is obtained, independent from the actual level of thrombin generation. In this way, APC resistance can be determined in the presence of antithrombotic therapy. The thrombotic tendency accompanying circulating lupus anticoagulant is readily displayed, in that, in these cases a prolongation of the lag time is seen but also an outspoken resistance to thrombomodulin [18].

\section{Anticoagulant drugs}

The aPTT is a poor means for measuring the heparin effect. We found that after heparin injection it was prolonged only in about one third of the samples in which the presence of heparin (unfractionated- or low molecular weight) could be demonstrated by its anti-factor Xa activity. Thrombin generation however was significantly diminished in over $80 \%$ of the cases (to be published). Oral anticoagulant treatment decreases thrombin generation, independent of the type and concentration ( $>5 \mathrm{pM}$ ) of thromboplastin used. Because proteins $\mathrm{S}$ and $\mathrm{C}$ are vitamin $\mathrm{K}$ dependent, one observes a certain APC resistance upon addition of Thrombomodulin. Unlike clotting times, the thrombogram quantitatively reflects the combined action of heparins and oral anticoagulation.

We conclude that the Thrombogram can be conveniently used to diagnose hyper- and hypocoaguable states, either congenital, acquired or drug-induced in humans and animals and hence to monitor prophylactic or therapeutic therapy with antithrombotics and in general all drugs that influence the function of the clotting system and all diseased states characterised by malfunction of this system.

\section{References}

1 Hemker HC, Béguin S: Phenotyping the clotting system. Thromb Haemost 2000;84:747-51.

2 Macfarlane RG, Biggs R: A thrombin generation test. J Clin Pathol 1953;6:3-7.

3 Pitney WR, Dacie JA: simple method of studying the generation of thrombin in recalcified plasma. J Clin Pathol 1953;6:9-13.

4 Hemker HC: Thrombin generation, an essential step in haemostasis and thrombosis. In Haemostasis and Thrombosis: Bloom AL, Forbes CD, Thomas DP, Tuddenham EGD, (eds). Churchill Livingstone, 1994;477-490.

5 Beguin S, Kumar R, Keularts I, Seligsohn U, Coller BS, Hemker HC: Fibrin-dependent platelet procoagulant activity requires GPIb receptors and von Willebrand factor. Blood 1999;93:564-70.

6 Hemker HC, Béguin S: Method for determining the endogenous thrombin potential of plasma and blood. U.S.Patent 5,192,689. 1993

7 Hemker HC, Wielders S, Kessels H, Béguin S: Continuous registration of thrombin generation in plasma, its use for the determination of the thrombin potential. Thromb Haemost 1993;70:617-24.

8 Duchemin J, Pittet JL, Tartary M, Béguin S,
Gaussem P, Alhenc-Gelas M, Aiach M: A new assay based on thrombin generation inhibition to detect both protein $\mathrm{C}$ and protein $\mathrm{S}$ deficiencies in plasma. Thromb Haemost 1994;71:331-8.

9 Curvers J, Thomassen MC, Rimmer J, Hamulyak K, van der Meer J, Tans G, Preston FE, Rosing J: Effects of hereditary and acquired risk factors of venous thrombosis on a thrombin generation-based APC resistance test. Thromb Haemost 2002;88:5-11

10 Tyler HM: Fibrin crosslinking demonstrated by thromboelastography: Thromb Diath Haemorrh 1969;22:398-400.

11 Mosesson MW, Kaminski M: The acceleratory effect of thrombin on fibrin clot assembly. Blood Coagul Fibrinolysis 1990; 1: 475-8

12 Beguin S, Lindhout T, Hemker HC: The effect of trace amounts of tissue factor on thrombin generation in platelet rich plasma, its inhibition by heparin. Thromb Haemost, 1989. 61(1): 25-9

13 Hemker HC, Giesen PL, Ramjee M, Wagenvoord R, Béguin S: The thrombogram: monitoring thrombin generation in platelet rich plasma. Thromb Haemost 2000;83:589-91.
14 Hemker HC, Giesen P, AlDieri R, Regnault V, de Smed E, Wagenvoord R, Lecompte TL, Béguin S. The Calibrated Automated Thrombogram, in platelet rich- and platelet poor plasma, applications in Clinical Diagnosis and Control of Antithrombotic Therapy. J Pathophysiol Haem Thromb: in press.

15 Hemker HC, Beguin S: Thrombin generation in plasma: its assessment via the endogenous thrombin potential. Thromb Haemost, 1995. 74: $134-8$.

16 Al Dieri R, Peyvandi F, Santagostino E, Giansily M, Mannucci PM, Schved JF, Béguin $\mathrm{S}$, Hemker HC: The thrombogram in rare inherited coagulation disorders: its relation to clinical bleeding. Thromb Haemost 2002; 88: 576-82.

17 P. M. Mannucci: The measurement of multifactorial Thrombophilia. Thromb. Haemost. 2002; 88:1-2.

18 Regnault V, Béguin S, Wahl D, de Maistre E, Hemker HC, Lecompte T: Thrombinography shows acquired resistance to activated protein $\mathrm{C}$ in patients with lupus anticoagulants. Thromb Haemost 2003; in press. 